\title{
OP03
}

\section{THE VISIBILITY OF THE PHANTOM ARRAY EFFECT UNDER OFFICE LIGHTING CONDITION}

\author{
Wang Lili et al.
}

DOI 10.25039/x46.2019.OP03

from

CIE x046:2019

Proceedings

of the

29th CIE SESSION

Washington D.C., USA, June 14 - 22, 2019

(DOI 10.25039/x46.2019)

The paper has been presented at the 29th CIE Session, Washington D.C., USA, June 14-22, 2019. It has not been peer-reviewed by CIE.

(C) CIE 2019

All rights reserved. Unless otherwise specified, no part of this publication may be reproduced or utilized in any form or by any means, electronic or mechanical, including photocopying and microfilm, without permission in writing from CIE Central Bureau at the address below. Any mention of organizations or products does not imply endorsement by the CIE.

This paper is made available open access for individual use. However, in all other cases all rights are reserved unless explicit permission is sought from and given by the CIE.

CIE Central Bureau

Babenbergerstrasse 9

A-1010 Vienna

Austria

Tel.: +4317143187

e-mail: ciecb@cie.co.at

www.cie.co.at 


\title{
THE VISIBILITY OF THE PHANTOM ARRAY EFFECT UNDER OFFICE LIGHTING CONDITION
}

\author{
Wang, L.L. ${ }^{1 *}$, Tu, Y. ${ }^{1}$, Cheng. S.L. ${ }^{1}$, Yu, X.L. ${ }^{1}$, Perz, M. ${ }^{2}$ Sekulovski, D. ${ }^{2}$ \\ ${ }^{1}$ School of Electronic Science and Engineering, Southeast University, Nanjing, CHINA \\ 2 Signify Research, NETHERLANDS \\ *wangll@seu.edu.cn
}

DOI 10.25039/x46.2019.OP03

\begin{abstract}
The phantom array effect, also known as ghosting, is one of the temporal light artefacts caused by temporally modulated light systems. In this study, the visibility of the phantom array effect under indirect viewing office lighting conditions was tested to verify whether it can cause a problem when making saccades across words during reading. Results show that, under a very critical condition of a white target on a black surface, the phantom array effect is easily perceived, especially at the intermediate frequency of $600 \mathrm{~Hz}$. But under the normal reading condition of a black target on a white surface, the phantom array effect becomes very difficult to detect.
\end{abstract}

Keywords: Visual perception, phantom array effect, ghosting, temporal light artefacts

\section{Introduction}

Light emitting diodes (LEDs) are the dominant general purpose lighting technology nowadays, thanks to their high performance and low power consumption. One of the most attractive advantages is the fast response of the light output to changes in the driving current, which enables the easy creation of dynamic light effects. However, the changes of luminance caused by these dynamic light effects could produce some unwanted spatio-temporal changes in the viewer's perception of the environment, which are termed temporal light artefacts (TLAs). TLAs could not only impair lighting quality, but also increased the risk of having a negative impact on human's body (Stone,1992). Thus, it's very important to better understand the visibility of the TLAs and to prevent their occurrence.

There are three kinds of TLAs, named flicker, stroboscopic effect, and the phantom array effect. The phantom array effect, also known as ghosting, is the focus of this study. The phantom array effect is defined as "change in perceived shape or spatial positions of objects, induced by a light stimulus the luminance or spectral distribution of which fluctuates with time, for a nonstatic observer in a static environment." (CIE TN 006:2016)

Until now, most studies focused on the visibility of this phenomenon under direct view conditions, i.e. when observing the light source directly. In such viewing conditions, the phantom array effect is visible at frequencies above the critical flicker frequency, i.e. $80 \mathrm{~Hz}$, and is more readily perceived for light source with high luminance and a sharp edge (Vogels I,2012; Lee C S, 2016), creating a high contrast between the target and the background. Besides, the phantom array effect is reported to be less visible at higher frequencies (Vogels I, 2012; Roberts J E, 2013; Wang L, 2017). However, our recently study reveals that, the visibility of this effect depends on frequency, following a U-shape form, with the effect being most visible at around $600 \mathrm{~Hz}(\mathrm{Yu}$ $X L, 2018)$. This study also presents that, the phantom array effect is more visible when the light waveform is square, compared to sinusoidal wave, and blue light results in less visible effect, compared to red and white light.

However, some research has suggested that the phantom array effect also might be perceived during reading, i.e. under indirect viewing conditions, when the light source generating temporally modulated light is not in the observer's field of view. It has been argued that under such conditions making saccades across words can result in a visible phantom array effect 
which might interfere with reading. Thus, it's important to also study the phantom array effect under indirect viewing office lighting condition, and to verify whether it can cause a problem during reading.

In this paper, we present two perception experiments conducted under office lighting conditions, in which we studied the influence of different parameters: contrast of the pattern, temporal modulation frequency, and illumination level on the visibility of the phantom array effect. The results provide information about the visibility of the phantom array effect in applications with lighting conditions similar to offices, such as home and hospitability.

\section{Experimental design}

A typical office luminaire equipped with LEDs was mounted in a room. The voltage of the LEDs was controlled by a programmable waveform generator via a computer. The relation between voltage and output light intensity was measured to transform the desired intensity into the required voltage. A regular table was placed right under the luminaire, with the height of $75 \mathrm{~cm}$ above the floor.

In the first experiment, the table was covered with a black surface, and a white paper strip that served as the target, was placed in the center of it. The strip had the width of $0.2 \mathrm{~cm}$ and the length of $2.5 \mathrm{~cm}$. During the experiment, the averaged illumination on the table was about 250 $\mathrm{Ix}$, and the Michelson contrast was around 0.93 between the target and the background. Two fixation points were positioned at both the left edge and right edge of the desk, to generate a saccade amplitude of about 50 degrees. During the experiment, participants were instructed to make saccades over the white target by fixating alternatively at each of the two fixation points. Fig. 1 shows the experimental set-up.

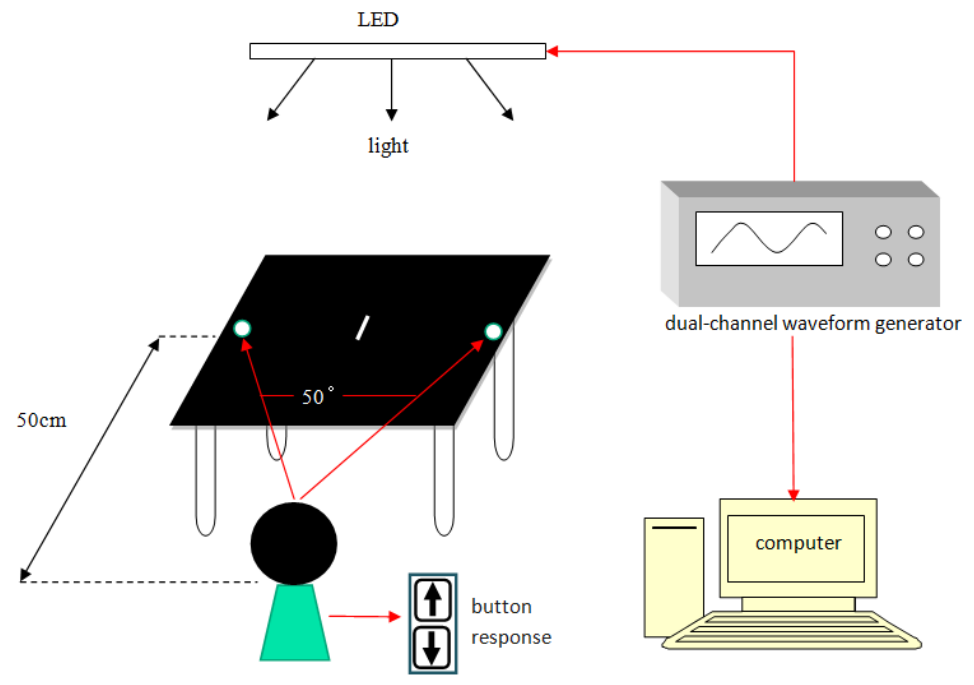

Figure 1 - The experimental set-up with the desk having black surface and a white target strip on it.

To test the visibility of the phantom array effect, sinusoidal waveforms with 3 different frequencies, i.e. $100 \mathrm{~Hz}, 600 \mathrm{~Hz}$ and $1200 \mathrm{~Hz}$ with a series of 5 different modulation depth were generated to modulate the light output in varying degrees. We conducted pilot experiments to ensure the selected modulation depths were distributed within a reasonable range. With $100 \mathrm{~Hz}$ and $1200 \mathrm{~Hz}$, the modulation depths of $20 \%, 30 \%, 50 \%, 70 \%$ and $100 \%$ were selected. Since the pilot participants were more sensitive at $600 \mathrm{~Hz}$, and a modulation depth of $30 \%$ could be fully detected in the pilot experiment, 5 lower modulation depths were selected for this particular frequency, i.e. $8 \%, 10 \%, 12 \%, 15 \%$ and $20 \%$. In total, there were 15 stimuli (= 5 modulation depth * 3 frequency).

A 2-Alternative-Forced-Choice (2AFC) methodology was adopted in the experiment. During the experiment, participants were exposed to a number of trials. Each trial consisted of two light 
conditions: constant light output (DC) and modulated light (the stimulus light). The table was first illuminated by one of the two light conditions for 5 seconds, then the light turned off for 3 seconds, followed by the other light condition. Participants were instructed to constantly make eye saccades and to point out in which light condition they perceived the effect. For each of the 15 stimuli, ten repetitions were executed, constituting 150 trials in total. To limit the experiment duration and avoid visual fatigue and mental exhaustion, we divided the experiment into 3 sessions according to the three frequencies. Thus, we got 50 trials in each session, which took about 15 minutes for a single participant. In total, 20 subjects participated in this experiment, 10 males and 10 females, with age ranged between 22 and 26 .

To measure the visibility of the phantom array effect under a more normal office lighting condition, instead of the very critical condition tested in the first experiment, an inverse contrast pattern was presented: the table was covered with a white surface and a black paper strip that served as the target was placed in the center of it, yielding a Michelson contrast of around 0.93. We performed a pilot study which revealed that the phantom array effect was almost imperceptible. Thus, only the most sensitive situation obtained in the first experiment, i.e. sine wave with the frequency of $600 \mathrm{~Hz}$ at full modulation depth, was used as the test waveform in the second experiment. In addition, two illumination levels were tested in separate sessions. In session 1, the average illumination measured on the table was around $250 \mathrm{~lx}$, and in session 2 it was about $500 \mathrm{~lx}$. The other set-up and the procedure was the same as in the first experiment. All of the 20 participants from experiment 1 joined the first session, and 14 of them joined the second session.

\section{Results and Discussions}

The number of times the phantom array effect was correctly detected was counted and averaged for each stimulus and participant.

Results of the first experiment are presented in Fig. 2, with error bars representing the 95\% confidence intervals. Results show that, for all the three tested frequencies, the phantom array effect is more visible, i.e. has higher detection rate, at a higher modulation depth. Since the 2AFC methodology was adopted, the averaged visibility threshold could be deduced from the modulation depth corresponding to $75 \%$ of the correctly detection rate. The threshold was around $26 \%, 14 \%$ and $46 \%$ modulation depth for $100 \mathrm{~Hz}, 600 \mathrm{~Hz}$ and $1200 \mathrm{~Hz}$, respectively. It is notable that, the visibility threshold measured for the sine wave at the frequency of $600 \mathrm{~Hz}$ was lower than the threshold at $100 \mathrm{~Hz}$ and $1200 \mathrm{~Hz}$. This indicates that the phantom array effect is more readily visible at intermediate frequency of $600 \mathrm{~Hz}$, compared to the lower (100 $\mathrm{Hz}$ ) or higher frequencies $(1200 \mathrm{~Hz})$. This result corresponds to the U-shape sensitivity curve obtained in our previous study with direct viewing condition.

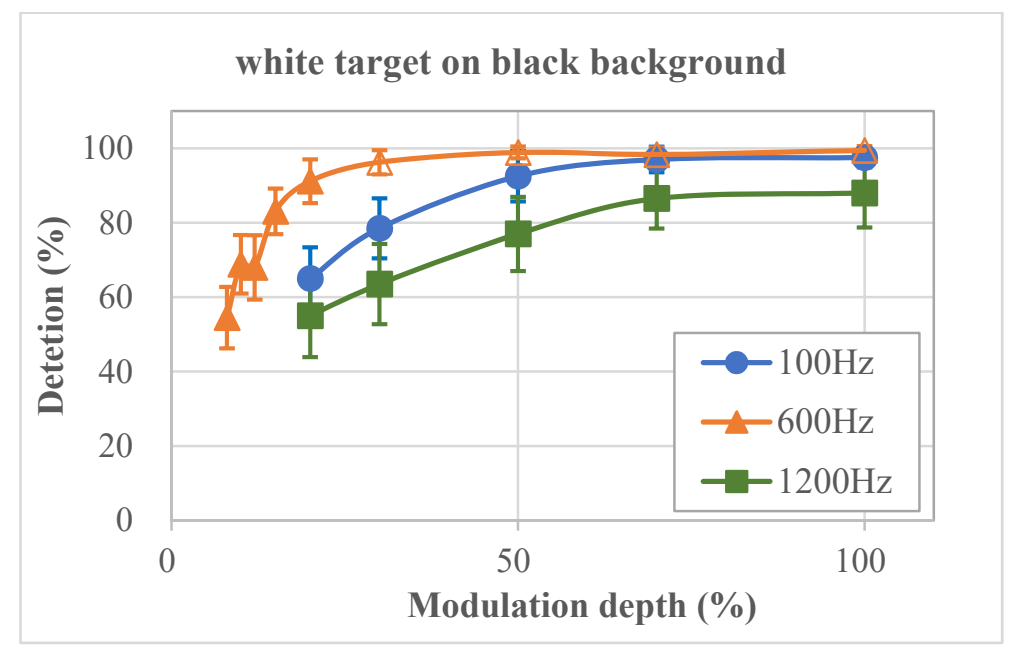

Figure 2 - Percentage of correct detections in the first experiment, as a function of modulation depth, for separate frequency (The hollow triangles representing the results of 6 participants obtained in the pilot experiment.) 
Averaged detection rate together with the $95 \%$ confidential interval of the second experiment are presented in Fig. 3. Results show that the averaged probability of detection is about $70.5 \%$ at $250 \mathrm{Ix}$ and $70 \%$ at $500 \mathrm{Ix}$. A large variation among participants was found. An independentsample t-test showed that there was no significant difference between these two illumination levels. As in a $2 \mathrm{AFC}$ method that was used, a $75 \%$ detection corresponds to a visibility threshold, the results indicate that most subjects cannot detect the phantom array effect under the condition with a black target on a white background. Besides, the illumination level within the range of $250 \mathrm{Ix}$ and $500 \mathrm{Ix}$ has no influence on the visibility.

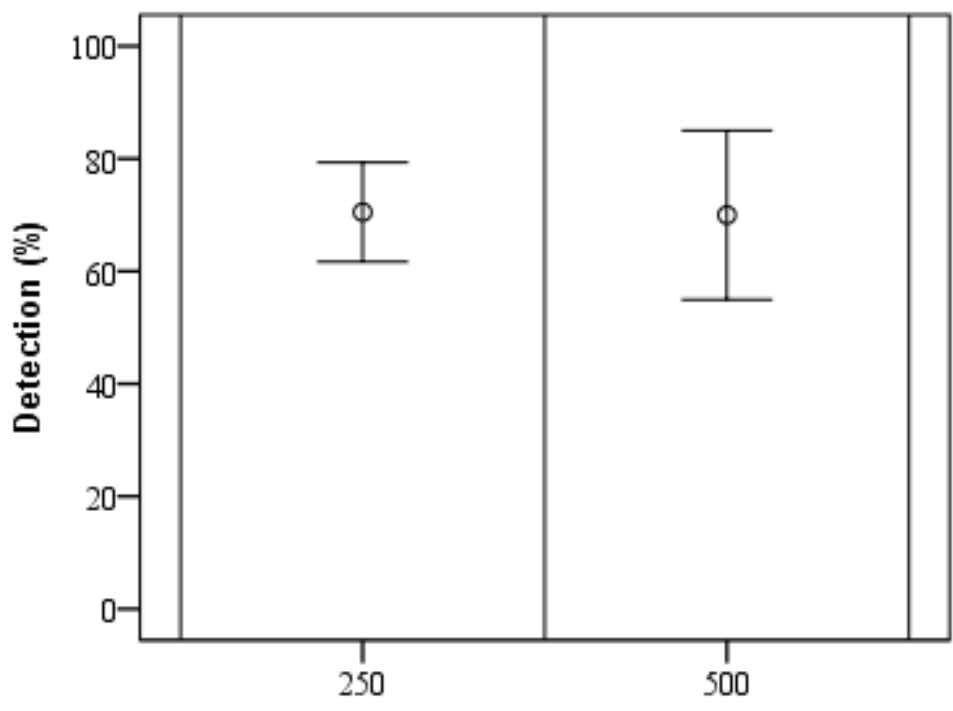

Illumination level (lux)

Figure 3 - Percentage of correct detections in the second experiment for illumination levels

\section{Conclusions}

This paper investigates the influence of contrast pattern, frequency, and illumination level on the visibility of the phantom array effect. The results obtained in this study generally show that the phantom array phenomenon could have some effect even though participants do not look at the light source directly, especially when they are looking at a white target on a black background. People are more sensitive to the effect at intermediate frequency levels (around $600 \mathrm{~Hz}$ ) compared to the lower or higher frequencies. This result corresponds well to the Ushape dependency of frequency to the visibility of the phantom array effect, obtained in our previous study with direct viewing condition.

However, if a participant is looking at a black target on white background, i.e. just like during normal reading, this effect is generally not visible, no matter whether the illumination level is high (around 500 lux) or low (around 250 lux). Furthermore, the size and corresponding speed of the saccades we used in the experiment were much larger than the usual sizes and speeds during normal reading. Both of these facts indicate that the phantom array effect should not be a problem during reading normal text.

As the maximal modulation possible for sinusoidal modulations at $600 \mathrm{~Hz}$ produced effects very close to the visibility threshold, further studies are needed to better measure the thresholds. One possible approach is to use square waveforms at different frequencies and with low duty cycles.. 


\section{Acknowledgements}

The authors would like to thank Dr. Kees Teunissen from Signify for his discussion during this study. And we are also grateful to all the volunteers for participating in the subjective experiments.

\section{References}

CIE TN 006:2016. Visual Aspects of Time-Modulated Lighting Systems - Definitions and Measurement Models.

LEE C S, PARK S W, et al. 2016. Effect of colour and luminance intensity on the phantom array. Proceedings of CIE, 2016: 232-240.

ROBERTS J E, WILKINS A J. 2013. Flicker can be perceived during saccades at frequencies in excess of $1 \mathrm{kHz}$. Lighting Research \& Technology, 45(1): 124-132.

STONE PT. 1992. Fluorescent lighting and health. Lighting Research \& Technology. 24(2), 5561

VOGELS I, HERNANDO I. Effect of eye movements on perception of temporally modulated light. 2012. Proceedings of Experiencing Light.

WANG LL, TU Y, LIU L, WANG JX, PERZ M and TEUNISSEN C. 2017. Phantom Array Effect of LED Lighting. Digest of SID 2017, 48(1): 1804-1807

YU XL, WANG LL, et.al. 2018. Influence of Frequency, waveform and colour on the visibility of the phantom array effect. Proceedings of CIE, 2018. 